
allemande

48-1 | 2016

Les expériences coloniales allemandes : échanges, transferts, circulations

\title{
Une démocratisation des politiques sociales d'entreprise? Le Volkswagenwerk à Wolfsburg et Automobiles Peugeot à Sochaux 1944-1980
}

\section{Ute Engelen}

\section{(2) OpenEdition}

\section{Journals}

Édition électronique

URL : https://journals.openedition.org/allemagne/345

DOI : 10.4000/allemagne.345

ISSN : 2605-7913

Éditeur

Société d'études allemandes

Édition imprimée

Date de publication : 15 juin 2016

Pagination : 159-177

ISSN : 0035-0974

Référence électronique

Ute Engelen, « Une démocratisation des politiques sociales d'entreprise ? Le Volkswagenwerk à Wolfsburg et Automobiles Peugeot à Sochaux 1944-1980", Revue d'Allemagne et des pays de langue allemande [En ligne], 48-1 | 2016, mis en ligne le 13 décembre 2017, consulté le 18 mai 2021. URL http://journals.openedition.org/allemagne/345 ; DOI : https://doi.org/10.4000/allemagne.345 


\section{Une démocratisation des politiques sociales d'entreprise? Le Volkswagenwerk à Wolfsburg et Automobiles Peugeot à Sochaux 1944-1980*}

- Ute Engelen**

Au croisement de l'entreprise et de la société, la politique sociale des établissements industriels est un objet de recherche stimulant. En contradiction avec l'hypothèse de sa perte d'importance due à l'essor de la réglementation ${ }^{(1)}$ et des politiques publiques, beaucoup d'entreprises ont remanié et même élargi leurs systèmes sociaux après la Seconde Guerre mondiale. Plusieurs acteurs de la politique sociale d'entreprise ont gagné en importance, notamment les représentants du personnel et des syndicats, les salariés et l'État ${ }^{(2)}$. Alors que les politiques sociales d'entreprise au XIX ${ }^{\mathrm{e}}$ siècle ont été largement étudiées par les historiens, celles d'après 1945 n'ont été explorées que

* Cet article présente une synthèse des résultats de la thèse publiée sous le titre Demokratisierung der betrieblichen Sozialpolitik? Das Volkswagenwerk in Wolfsburg und Automobiles Peugeot in Sochaux 1944-1980 en 2013 par l'éditeur Nomos. Je tiens à remercier particulièrement Catherine Maurer, Lisa Börner, Patrick Fridenson, Hervé Joly et Nadège Vézinat pour leurs suggestions constructives, mais j'assume la responsabilité de toute erreur.

** Collaboratrice scientifique à l'Institut für Geschichtliche Landeskunde an der Universität Mainz e.V. et au Stadthistorisches Museum Mainz.

1 Jean-Claude Daumas, "Les politiques sociales des entreprises en France 1880-1970», in: Hélène Fréchet (dir.), Industrialisation et sociétés en Europe occidentale de 1880 à 1970, Paris, Éditions du Temps, 1997, p. 105-125, ici p. 122; Günther ScHulz, «Betriebliche Sozialpolitik in Deutschland seit 1850 », in: Hans PонL (dir.), Staatliche, städtische, betriebliche und kirchliche Sozialpolitik vom Mittelalter bis zur Gegenwart, Stuttgart, Steiner, 1991, p. 137-176, ici p. 175.

2 Pascale Quincy-Lefebvre, «Le système social Michelin de 1945 à 1973 ou l'épuisement d'un modèle », in: André Gueslin (dir.), Les hommes du pneu. Les ouvriers Michelin à Clermont-Ferrand de 1940 à 1980, Paris, Les Éditions de l’Atelier/Les Éditions Ouvrières (Collection Mouvement Social), 1999, p. 93-219, ici p. 216; G. Schulz, «Betriebliche Sozialpolitik in Deutschland» (note 1), p. 164. Pour le développement de la participation ouvrière, voir Michel Pigenet, Danielle Tartakowsky (dir.), Histoire des mouvements sociaux en France de 1814 à nos jours, Paris, La Découverte, 2012, et plus spécialement Georges Mouradian (dir.), L'enfance des comités d'entreprise, Roubaix, CAMT, 1997. 
récemment ${ }^{(3)}$. Souvent, les recherches privilégient des points de vue économico-théoriques et d'histoire d'entreprise générale ${ }^{(4)}$, ce qui néglige la spécificité de l'engagement d'entreprise après la Seconde Guerre mondiale.

Dans cet article, nous avons fait le choix de mettre au premier plan la transformation de la politique sociale d'entreprise pendant la reconstruction, les Trente Glorieuses et les crises pétrolières. Les politiques sociales du Volkswagenwerk ${ }^{(5)}$ et des Automobiles Peugeot ${ }^{(6)}$ n’ont été traitées jusqu'ici que dans des contextes plus larges. Ces

3 Des exceptions sont Serge Bonnet (dir.), L’homme du fer. Mineurs de fer et ouvriers sidérurgistes lorrains, 3 vol., Nancy et al., Presses Universitaires de Nancy/Éditions Serpenoise, 1982-1984; J.-C. DAUMAS, «Les politiques sociales» (note 1); A. GuesLin (dir.), Les hommes du pneu (note 2); Gérard VINDT, Les hommes de l'aluminium. Histoire sociale de Pechiney 1921-1973, Paris, Les Éditions de l'Atelier/Les Éditions Ouvrières, 2006. De nouvelles recherches depuis 2011: Almuth Bartels, Monetarisierung und Individualisierung. Historische Analyse der betrieblichen Sozialpolitik bei Siemens (1945-1989), Stuttgart, Steiner (Beiträge zur Unternehmensgeschichte, 32), 2013; Rüdiger GerLACH, Betriebliche Sozialpolitik im historischen Systemvergleich. Das Volkswagenwerk und der VEB Sachsenring von den 1950 er bis in die 1980er Jahre, Stuttgart, Steiner (Vierteljahrschrift für Sozial- und Wirtschaftsgeschichte, Beiheft 227), 2014; Nina KLEINÖDER, Unternehmen und Sicherheit. Strukturen, Akteure und Verflechtungsprozesse im betrieblichen Arbeitsschutz der westdeutschen Eisen- und Stahlindustrie nach 1945, Stuttgart, Steiner (Vierteljahrschrift für Sozial- und Wirtschaftsgeschichte, Beiheft 234), 2015. La thèse de Stephanie Hagemann-Wilholt sur les bilans sociaux d'entreprise doit paraître.

4 Par exemple J.-C. Daumas, «Les politiques sociales» (note 1); G. Vindt, Les hommes de l'aluminium (note 3).

5 Nous n'évoquerons ici que des ouvrages récents et significatifs pour les deux constructeurs. Heidrun Edelmann, Heinz Nordhoff und Volkswagen. Ein deutscher Unternehmer im amerikanischen Jahrhundert, Göttingen, Vandenhoeck \& Ruprecht, 2003, p. 151-167; Hans Mommsen, Manfred Grieger, Das Volkswagenwerk und seine Arbeiter im Dritten Reich, Düsseldorf, ECON, 1996; Markus LupA, Spurwechsel auf britischen Befehl. Der Wandel des Volkswagenwerks zum Marktunternehmen 1945-1949, Wolfsburg, Volkswagen AG, 2010; Hedwig Richter, Ralf Richter, Die Gastarbeiter-Welt. Leben zwischen Palermo und Wolfsburg, Paderborn, Schöningh, 2012; Ralf RichteR, «Die Währungs- und Wirtschaftsreform 1948 im Spiegel unternehmerischer Personalpolitik. Volkswagen 1945 bis 1950 », Zeitschrift für Unternehmensgeschichte, vol. 48 (2003), p. 215-238; ID, Ivan Hirst. Britischer Offizier und Manager des Volkswagenaufbaus, Wolfsburg, Volkswagen AG, 2003; Steven TollidAY, «Enterprise and State in the West German Wirtschaftswunder. Volkswagen and the Automobile Industry 19391962 », Business History Review, vol. 69 (1995), p. 273-350 ; Volker WellHöner, "Wirtschaftswunder" - Weltmarkt - westdeutscher Fordismus. Der Fall Volkswagen, Münster, Westfälisches Dampfboot, 1996.

6 Stéphane Beaud, Michel Pialoux, Retour sur la condition ouvrière. Enquête aux usines Peugeot de Sochaux-Montbéliard, Paris, Fayard, 1999; Robert Belot, Pierre Lamard, Peugeot à Sochaux. Des hommes, une usine, un territoire, Panazol, Lavauzelle, 2007; Yves CoHen, "L'invention des techniciens sociaux. Du commandement social après juin $1936 \mathrm{chez}$ Peugeot», Actes de la recherche en sciences sociales, $\mathrm{n}^{\circ} 114$ (1996), p. 30-43 ; Marcel DurAND, Grain de sable sous le capot. Résistance et contre-culture ouvrière. Les chaînes de montage de Peugeot (1972-2003) (1990), Marseille, Agone, 2006; Jean-Pierre Durand, Nicolas Hatzfeld, La chaîne et le réseau. Peugeot-Sochaux, ambiances d'intérieur, Lausanne, Page deux, 2002; Nicolas Hatzfeld, Les gens d'usine. 50 ans d'histoire à Peugeot-Sochaux, Paris, Les Éditions de l'Atelier/Les Éditions Ouvrières, 2002; ID., "L'usine automobile des années 1950, une usine à l'américaine? Le cas de Peugeot-Sochaux», in: André KAspi (dir.), Les relations franco-américaines dans le domaine de la technologie. Colloque organisé à la Fondation Singer-Polignac le 26 novembre 1998, Paris, Paillart, 2000, p. 39-58; Jean-Louis Loubet, La Maison Peugeot, Paris, Perrin, 2009; ID., «PSA Peugeot Citroën, 1973-1992, histoire d'un groupe automobile dans la crise», Actes du GERPISA, n 10 (1994), p. 109-147; ID., Automobiles Peugeot. Une réussite industrielle 1945-1974, Paris, Economica, 1990. 
deux entreprises sont comparables pour plusieurs raisons. Actives dans la construction automobile généraliste, l'une, Peugeot, était une entreprise familiale, l'autre, Volkswagen, une entreprise publique, mais toutes les deux étaient gérées comme des entreprises privées ${ }^{(7)}$. Leurs usines principales installées respectivement à Wolfsburg et à Sochaux étaient situées dans des régions relativement peu peuplées, ce qui les a amenées à offrir des prestations sociales élevées ${ }^{(8)}$. Leur comparaison permet d'éclairer non seulement les facteurs qui ont influencé leur politique sociale, mais aussi le rôle de l'environnement national. Le fait que les deux entreprises sont issues de la même branche de production réduit le risque d'une surévaluation de leurs spécificités. Nos questions étaient les suivantes: dans quelle mesure les aspects de la politique sociale d'entreprise ont évolué? Quels sont les points communs et les divergences entre Volkswagen et Peugeot dans ce domaine et sur quels facteurs sont-ils fondés? Dans quelle mesure certains acteurs ont marqué l'engagement social des entreprises et quel rôle incombe aux facteurs structurels?

Plusieurs approches théoriques tentent d'expliquer ce qui conduit les entreprises à pratiquer une politique sociale. L'ancien débat sur le paternalisme a perdu de sa force explosive ${ }^{(9)}$. Selon certaines définitions ${ }^{(10)}$ et de notre point de vue, Volkswagen et Peugeot ne peuvent pas être considérées comme paternalistes en raison de leur taille. En outre, les prestations sociales étaient attribuées par l'intermédiaire du département du personnel et de plus en plus marquées par l’influence des managers. Par ailleurs, l'approche paternaliste s'intéresse principalement aux motivations de la politique sociale, mais elle surestime également ses effets ${ }^{(11)}$. Dans la recherche économique et les études historiques récentes, des aspects rationnels comme le manque de main-d'œuvre sont mis en avant pour justifier l'engagement social des entreprises ${ }^{(12)}$.

7 Günther Косн, Arbeitnehmer steuern mit. Belegschaftsvertretung bei VW ab 1945, Cologne, BundVerlag, 1987, p. 139; J.-L. Loubet, Citroën, Peugeot, Renault et les autres. Soixante ans de stratégies, Paris, Le Monde-Éditions, 1995, p. 542.

8 Cf. Automobiles Peugeot (AP), Rapport annuel 1952, p. 10.

9 Jean Fombonne, Personnel et DRH. L'affirmation de la fonction Personnel dans les entreprises (France, 1830-1990), Paris, Vuibert, 2001, p. 167; Patrick FrIDENSON, «La subordination dans le travail. Les questions de l'historien», in: Jean-Pierre Chauchard, Anne-Chantal Hardy-Dubernet (dir.), $\mathrm{La}$ subordination dans le travail. Analyse juridique et sociologique de l'évolution des formes d'autonomie et de contrôle dans la relation de travail, Paris, La Documentation française, 2003, p. 59-69, ici p. 61.

10 Martin Fiedler, «Betriebliche Sozialpolitik in der Zwischenkriegszeit. Wege der Interpretation und Probleme der Forschung im deutsch-französischen Vergleich», Geschichte und Gesellschaft, vol. 22 (1996), p. 350-375, ici p. 353; Thomas Welskopp, «Betriebliche Sozialpolitik im 19. und frühen 20. Jahrhundert. Eine Diskussion neuerer Forschungen und Konzepte und eine Branchenanalyse der deutschen und amerikanischen Eisen- und Stahlindustrie von den 1870er bis zu den 1930er Jahren", Archiv für Sozialgeschichte, vol. 34 (1994), p. 333-374, ici p. 342.

11 J.-C. Daumas, «Les politiques sociales» (note 1), p. 105; T. Welskopp, «Betriebliche Sozialpolitik im 19. und frühen 20. Jahrhundert» (note 10), p. 336 sq.

12 Susanne Hilger, Sozialpolitik und Organisation. Formen betrieblicher Sozialpolitik in der rheinischwestfälischen Eisen- und Stahlindustrie seit der Mitte des 19. Jahrhunderts bis 1933, Stuttgart, Steiner, 1996, p. 37; Alain Leménorel, «Du paternalisme à la culture d'entreprise», in: Dominique BARJOT (dir.), Le travail à l'époque contemporaine, Paris, Éditions du CTHS, 2005, p. 173-186; Anne NieberDING, Unternehmenskultur im Kaiserreich. J. M. Voith und die Farbenfabriken vorm. Friedr. Bayer und Co., Munich, Beck, 2003; Sylvie SCHWEITzer, «"Paternalismes” ou pratiques sociales?», in: ID. (dir.), 
Ces approches de la nouvelle économie institutionnelle soulignent le caractère d'investissement d'une prestation sociale, visant une plus grande productivité ou une minimisation des coûts. Néanmoins, cet effet positif est mis en doute par des études empiriques ${ }^{(13)}$ et ces hypothèses négligent aussi le rôle des acteurs impliqués, les entrepreneurs exceptés.

Au plus tard depuis l'ordonnance du 22 février 1945 sur les comités d'entreprise (CE) et le Betriebsverfassungsgesetz de 1952, les chefs d'entreprise étaient tenus de négocier avec les CE et les syndicats. Ainsi, il ne semble plus pertinent de rechercher les raisons de l'engagement social dans les entreprises industrielles, mais bien plutôt d'étudier les processus de décision, les formes de politique sociale et leurs effets réels ${ }^{(14)}$. Nous avons employé l'approche «micropolitique » ${ }^{(15)}$ pour éclaircir les situations de décision complexe relatives à la modification d'une prestation sociale. La valorisation des acteurs permet de nuancer l'idée d'une politique sociale statique. Néanmoins, la stabilité de cette politique, favorisée par la dépendance à l'égard du chemin déjà parcouru (path dependency), la judiciarisation et la pesanteur des habitudes, ne doit pas être sousévaluée ${ }^{(16)}$, car les acteurs ne peuvent agir que dans un champ de décision restreint, ne disposant que d'informations limitées. Le concept d'embeddedness lancé par Mark Granovetter ${ }^{(17)}$ complète l'approche micropolitique en mettant en avant l'importance des relations sociales et des facteurs structurels de l'action économique.

Nous avons consulté de nombreux documents issus des directions de Volkswagen, de Peugeot et de l'usine de Sochaux, des départements du personnel, des CE, de la CGT et de la CFDT; ces documents ont été complétés par des archives publiques. Nous avons également réalisé des entretiens semi-directifs avec des anciens salariés des entreprises, des militants et des cadres des départements sociaux et du personnel. Trois périodes ont été distinguées. Pour chacune d'entre elles, nous analyserons les catégories assistance, prestations économiques, politique d'infrastructure et loisirs.

Logiques d'entreprises et politiques sociales des XIX et $X X^{e}$ siècles, Villeurbanne, Programme RhôneAlpes pluriannuel de recherches en sciences humaines, 1993, p. 5-18.

13 Bernd FrICK, «Die Anreizwirkungen betrieblicher Sozialleistungen: Möglichkeiten und Grenzen», in: Winfried Sснмёнl (dir.), Soziale Sicherung zwischen Markt und Staat, Berlin, Duncker \& Humblot, 2000, p. 189-214, ici p. 212 sq. Différemment Bernard CAIlley, Politique sociale de l'entreprise et performance économique. La politique sociale désigne la manière dont l'employeur conçoit, organise et met en cuvre les relations de travail dans l'entreprise, Paris, Les Éditions d'organisation, 1996, p. 133.

14 T. Welskopp, «Betriebliche Sozialpolitik im 19. und frühen 20. Jahrhundert» (note 10), p. 349.

15 Tom Burns, «Micropolitics. Mechanisms of Institutional Change », Administrative Science Quarterly, vol. 6 (1961-62), p. 257-281. Voir aussi Karl Lauschke, Thomas Welskopp (dir.), Mikropolitik im Unternehmen. Arbeitsbeziehungen und Machtstrukturen in industriellen Großbetrieben des 20. Jahrhunderts, Essen, Klartext, 1994.

16 J.-C. Daumas, «Les politiques sociales» (note 1), p. 119 sq.; G. SCHulz, «Betriebliche Sozialpolitik in Deutschland» (note 1), p. 174.

17 Mark Granovetter, "Economic Action and Social Structure. The Problem of Embeddedness", American Journal of Sociology, vol. 91 (1985), p. 481-510. 


\section{Entre reconstruction et expansion - du milieu des années 1940 au début des années 1950}

Certaines continuités et transformations de la politique sociale d'entreprise ont été déterminées par la propre histoire des entreprises, leur régulation croissante et l'extension de la politique sociale publique. Des changements de structure et d'acteur ont également été importants, avec par exemple la généralisation des conventions collectives donnant un nouveau rôle aux syndicats. Les institutions représentatives du CE et du Betriebsrat $^{(18)}$ sont devenues obligatoires en France en 1945 et en RFA en 1952. Ces évolutions ont amené les partenaires sociaux à être davantage contraints au compromis.

À de nombreux égards, Peugeot en novembre 1944 et Volkswagen en avril 1945 ont maintenu leurs pratiques sociales, en particulier le poids de l'assistance de la période de guerre $^{(19)}$, et ceci malgré la gestion de l'entreprise allemande par le gouvernement militaire britannique entre 1945 et 1949. Mais déjà dans les années 1940, les deux entreprises avaient jeté les bases de nombreuses prestations sociales qui prirent de l'importance plus tard. Après un blocage de la progression des salaires, les rémunérations réelles étaient en forte augmentation depuis 1949. Mais les prestations d'assistance se développaient dans un sens contraire: la première période peut être considérée comme une période de transition entre un "approvisionnement total» des salariés jusqu'en 1948 et la conservation ensuite de prestations choisies, plutôt financières, comme le versement de secours.

Entre 1945 et 1951, Volkswagen et Peugeot augmentent considérablement leur production dans le cadre de la reconstruction - le constructeur allemand produit plus de 100000 voitures, Peugeot presque 81000 . En même temps, elles doublent quasiment leurs effectifs. Chaque salarié étant difficile à remplacer et indispensable pour une production donnée, les entreprises font le choix d'investir dans les prestations sociales. Chez Volkswagen, les charges "volontaires" s'élèvent à $25,5 \%$ de la masse salariale en 1949 et à $14,1 \%$ en 1951 (l'ensemble des charges sociales, charges volontaires incluses, s'élevant alors à 31,0\%). Chez Peugeot, les charges sociales atteignent $37 \%$ de la masse salariale en 1948 et $40,8 \%$ en 1950 .

Dans les premiers temps, ce sont l'approvisionnement et l'assistance qui ont la priorité, à l'exception de la politique d'infrastructure, car les besoins physiologiques de base des salariés ne sont pas assurés et les entreprises estiment devoir fournir ces prestations pour pouvoir fonctionner ${ }^{(20)}$. Elles ne les ont supprimées que lorsque les salariés n'en ont plus eu besoin ou lorsqu'elles ont été fournies par d'autres acteurs. Les CE ne s'y opposaient pas parce que les moyens financiers pouvaient être investis dans des prestations désormais davantage appréciées par les salariés (figure 1). Les cantines $^{(21)}$, les magasins Ravi de Peugeot et la vente de voitures avec une ristourne ont subsisté car leur fonction ne se limitait pas à l'approvisionnement.

18 Ses membres sont élus par les salariés d'un établissement. À la différence du CE, l'employeur n’y est pas représenté.

19 Mommsen/ Grieger, Das Volkswagenwerk und seine Arbeiter (note 5), p. 414.

20 Cf. aussi Karl Lauschke, Die Hoesch-Arbeiter und ihr Werk. Sozialgeschichte der Dortmunder Westfalenhütte während der Jahre des Wiederaufbaus 1945-1966, Essen, Klartext, 1999, p. 89.

21 Un numéro spécial du Mouvement Social est consacré aux cantines d'entreprise: Le Mouvement Social, $\mathrm{n}^{\circ} 247$ (2014), L'alimentation au travail depuis le milieu du XIX $X^{e}$ siècle. 


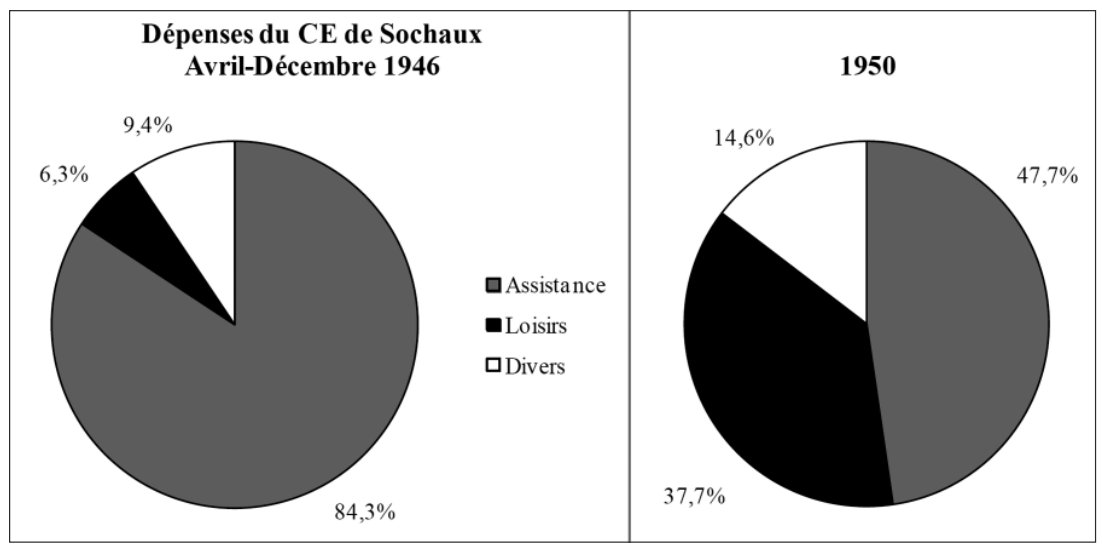

Figure 1: Rapport du Comité d'Établissement de Sochaux sur la gestion des œuvres sociales, 1.04.-31.12.1946, p. 11, et 1950, annexe Exploitation Générale, in : CE1, PV du CE.

Les prestations accordées pour des raisons économiques étaient supposées améliorer la satisfaction au travail, augmenter la productivité et réduire le turn-over. Une fois la pénurie de nourriture résolue, les entreprises devaient améliorer leur productivité. Elles ont ainsi systématisé leurs prestations financières. Le nombre de primes augmente, en particulier chez Peugeot ${ }^{(22)}$ qui établit une prime d'intéressement à partir de 1950. À partir de 1949, et jusqu'au début de la participation aux bénéfices en 1954, Volkswagen a décaissé chaque année une prime exceptionnelle ${ }^{(23)}$. En 1949, après Peugeot, elle a créé une assurance vieillesse et une assurance vie. Les deux entreprises récompensaient déjà leurs salariés pour leurs suggestions d'amélioration, mais un procédé leur garantissant un examen juste des idées et des primes plus intéressantes ont été introduites en 1949 chez Volkswagen et en 1951 chez Peugeot.

Tout comme l'assistance, la politique d'infrastructure s'est révélée essentielle afin de garantir la production. Sans les cars d'entreprise chez Peugeot ou les hébergements pour célibataires chez Volkswagen, les entreprises n'auraient pas pu employer autant de salariés. Néanmoins, de nombreux projets d'habitat ne se sont pas réalisés à cause du manque de matériel et de capitaux. Et la constitution d'un système de transport a été plus attractive pour les établissements, aussi longtemps qu'il y a eu suffisamment de salariés potentiels dans les environs. L’engagement des entreprises se limitait surtout à la construction de foyers pour célibataires et à la transformation de baraques et de dortoirs. Dans ce domaine, le CE de Sochaux a poursuivi une politique plus active que le Betriebsrat de Wolfsburg. Cependant, les représentants du personnel des deux usines avaient les mêmes difficultés à imposer leurs points de vue concernant l'attribution des logements, probablement parce que les directions concevaient cette dernière comme importante pour la production.

22 N. Hatzfeld, Les gens d'usine (note 6), p. 218. Voir aussi Yannick Lemarchand, «Règlement de la prime de production et de gestion. Berliet 1946 ", Entreprises et Histoire, n 79 (2015), p. 188-193.

23 Volkswagenwerk (VWW), Jahresbericht des Personalwesens 1952, p. 3, in: Unternehmensarchiv der Volkswagen Aktiengesellschaft (UVW), Z 69 n 701. 
L'engagement des entreprises dans le domaine des loisirs et des vacances était marqué d'abord par un objectif d'amélioration de la santé et d'entraînement physique, mais aussi de renforcement de la communauté des salariés et d'éducation de ces derniers. Il s'agit de l'un des rares secteurs où les politiques de Volkswagen et de Peugeot divergent. Ainsi, le CE de Sochaux a privilégié les colonies de vacances comme c'était de coutume ailleurs en France, tandis que Volkswagen a continué à offrir des congés surtout pour convalescence ou comme récompense. Alors que le CE de Sochaux soutenait l'expansion des loisirs, la direction de Volkswagen ne s'est guère engagée dans ce domaine. La différence des politiques est à mettre en relation avec l'influence divergente des représentants du personnel: un manager comme Heinrich Nordhoff ${ }^{(24)}$ souhaitait offrir des prestations sociales utiles à la production et un engagement dans les loisirs aurait pu rappeler les mesures Kraft durch Freude mises en place par le syndicat unique nazi. Le Betriebsrat ne possédait pas dans ce domaine de budget comparable à celui du CE français. Bien que les vacances soient un avantage pour les enfants comme pour les adultes ${ }^{(25)}$, les employés de Volkswagen ne pouvaient donc partir que sans leurs familles.

L'examen des politiques sociales dans la première période a montré que les deux entreprises étaient convaincues qu'il fallait satisfaire les besoins élémentaires de leurs salariés afin d'assurer leur emploi et leur productivité. Dès qu'une prestation ne semblait plus demandée ou appropriée pour garantir la production, elle était supprimée. La transition d'une économie de guerre vers une économie de paix a entraîné une division des fonctions sociales entre les entreprises, les marchés et les institutions publiques. Néanmoins, les entreprises n'ont pas renoncé à toute mesure extérieure à la production. La plupart des prestations sociales qui sont devenues plus importantes ultérieurement ont été établies avant les années 1950, même si elles restaient souvent alors à un stade expérimental. Par exemple, Volkswagen n'attribuait des prêts à la construction qu'à une petite partie de ses salariés.

Le Betriebsrat et le syndicat IG Metall à Wolfsburg avaient une influence assez faible et étaient rarement suivis par les autorités d'occupation britanniques ou la direction ${ }^{(26)}$. Notamment parce qu'il gérait les principales œuvres sociales, le CE de Sochaux avait davantage d'influence. En outre, ses impulsions orientaient les décisions des directions d'entreprise et d'usine. Deux exceptions à cela étaient l'attribution de primes et le financement des logements, pour lesquels les directions prenaient la plupart des décisions, tandis que le CE ne pouvait qu'en contester les conditions d'application.

24 Cf. Hervé Joly, Patrons d’Allemagne. Sociologie d’une élite industrielle 1933-1989, Paris, Presses de FNSP, 1998.

25 Georges Cazes, Françoise Potier, «Un “système vacancier” français?», in: Jean Viard et al. (dir.), La France des temps libres et des vacances, Gémenos, Éditions de l'Aube, 2002, p. 45-61, ici p. 47; Christine KeItz, Reisen als Leitbild. Die Entstehung des modernen Massentourismus in Deutschland, Munich, Deutscher Taschenbuch Verlag, 1997, p. 336.

26 Udo Riechert, Neubeginn im Schatten der NS-Tyrannei. Gewerkschaften und Betriebsräte in Braunschweig und Wolfsburg in den ersten Nachkriegsjahren, Braunschweig, Steinweg, 1987, p. 226. 


\section{Volume et diversification - du début des années 1950 au milieu des années 1960}

Des années 1950 aux années 1960, l'industrie automobile grandit de manière presque continue en France comme en RFA. Volkswagen augmente le volume de sa production de plus de huit fois entre 1952 et 1963, Peugeot de plus de trois fois. Ce «triomphe de la révolution automobile " ${ }^{(27)}$ n'est pas dû seulement à la production de masse "fordiste» ou à la transformation du système social public, mais aussi à l'établissement de conditions sociales appropriées dans les établissements industriels ${ }^{(28)}$. L'augmentation de la production se fonde surtout sur une hausse des effectifs des salariés. À Wolfsburg, le personnel augmente de $163 \%$ et s'élève finalement à 44000 salariés en 1963, tandis qu'à Sochaux, l'effectif double quasiment. Dans les deux cas, la progression repose surtout sur le recrutement de main-d'œuvre intérieure. La proportion des salariés étrangers chez Volkswagen n'excède pas $1 \%$ entre 1950 et 1960. À Sochaux, elle passe de $18,2 \%$ en 1948 à un niveau plancher de $5,3 \%$ en $1964^{(29)}$.

La politique sociale a été une condition sine qua non de l'expansion des effectifs et ainsi du succès économique des deux constructeurs automobiles pendant cette période. Davantage qu'auparavant, les établissements devaient se rendre attractifs auprès de nouveaux salariés, notamment parce que leur environnement géographique ne permettait plus une expansion suffisante en personnel ${ }^{(30)}$. Avant 1963, les charges sociales supplémentaires de Volkswagen sont passées à 17,8 \% de la masse salariale et l'ensemble des coûts sociaux a atteint 39,3\% de cette même masse salariale. Chez Peugeot, les dépenses sociales étaient de 39,4\% en 1959, mais sont passées à 31,2\% en 1966 après la restructuration de l'entreprise. Ceci était vraisemblablement une conséquence de l'augmentation des salaires et d'une réduction des prestations, avec l'arrêt de l'accord d'entreprise, ainsi que des dépenses de logement à la suite de la crise de 1964/65.

En ce qui concerne l'assistance matérielle, les deux entreprises se sont concentrées sur certains secteurs comme les cantines qu'elles ont modernisées en installant des distributeurs et des repas en libre-service conformément aux souhaits des salariés. En externalisant les cantines, Peugeot s'est désengagée des subventions croissantes pour les repas. Ce type de soutien matériel aux salariés a perdu en importance, en devenant de plus en plus superflu au fur et à mesure que les produits étaient plus accessibles et que les revenus augmentaient. Néanmoins, les entreprises ont maintenu des prestations dont dépendaient encore certains salariés, surtout les plus âgés. Toutefois, tandis que les dépenses pour secours immédiat diminuaient (figure 2), le montant des allocations plus universelles comme la prime pour charges de famille a progressé. Bien que l'assistance et l'approvisionnement en biens matériels ne soient plus des préalables à la production, les deux entreprises les percevaient encore comme des éléments indispensables à la qualité des relations sociales.

27 Jean-Pierre BArdou et al. (dir.), La révolution automobile, Paris, Albin Michel, 1977.

28 V. WellhöNER, "Wirtschaftswunder" - Weltmarkt-westdeutscher Fordismus (note 5), p. 308.

29 VWW, Jahresbericht 1961 des Vorstandsbereiches "Personal”, p. 1, in: UVW, Z 69 n 701 ; BELOT/ Lamard, Peugeot à Sochaux (note 6), p. 43, 67, 72 sq.; N. Hatzfeld, Les gens d'usine (note 6), p. 169 sq., p. 558.

30 AP, Rapport annuel 1952, p. 10. 


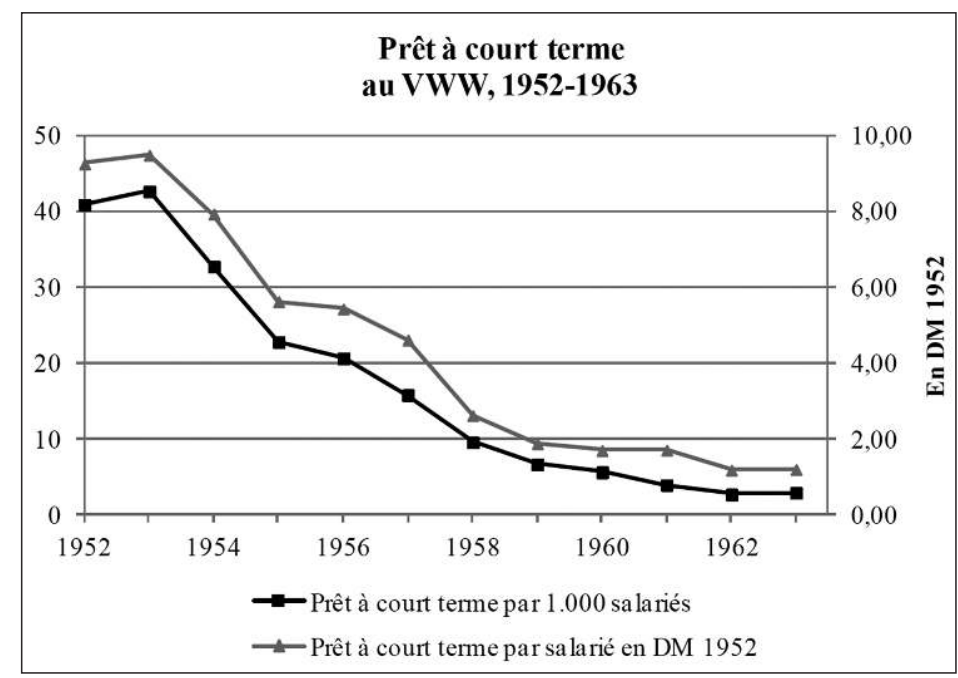

Figure 2: Les dépenses pour l'année 1960 ont été estimées.

VWW, Jahresberichte des Personalwesens 1952-1963, in: UVW, Z $69 \mathrm{n}^{\circ} 701$.

De plus en plus, Volkswagen et Peugeot accordaient des prestations soumises aux conditions économiques de l'entreprise. Peugeot ne s'est pas bornée pas à la prime de bilan introduite pour l'exercice 1950, elle a établi aussi une participation aux dividendes et aux bénéfices réinvestis. À partir de 1953, Volkswagen a mis en place une participation dont l'importance réelle par tête a triplé jusqu'en 1963. Les deux entreprises versaient aussi toujours des primes exceptionnelles. Peugeot a sensiblement augmenté les primes pour suggestion d'amélioration au début des années 1960, alors que Volkswagen ne l'a fait que quelques années plus tard. Les constructeurs étaient également disposés à financer des prestations à long terme comme les retraites complémentaires, mais ils se réservaient le droit de les remettre en cause si les conditions économiques évoluaient. Les récompenses pour années de services ont augmenté. En distribuant des primes, les constructeurs visaient à une participation des salariés au succès de l'entreprise et à une élévation de leur niveau de vie, sans pour autant s'engager durablement. Ces prestations dépendantes du succès de l'entreprise et de la performance des salariés leur semblaient nécessaires, mais elles relevaient aussi pour eux d'un «impératif de justice » ${ }^{(31)}$. Elles devaient aussi stimuler l'entrée à Volkswagen ou à Peugeot et étaient censées réduire le turn-over ${ }^{(32)}$. Pour cette raison, de nombreuses prestations dépendaient d'une certaine durée d'emploi.

Pour le domaine des infrastructures, la comparaison entre Volkswagen et Peugeot montre que les politiques des deux établissements évoluaient de manière convergente. Depuis les années 1950, les deux entreprises préféraient le financement d'HLM à

31 Heinrich Nordhoff, Reden und Aufsätze. Zeugnisse einer Ära, ECON, Düsseldorf et al., 1992, p. 212.

32 Entretien avec Gilbert Motelier et Michel Tridard le 14.11.2008 et avec Werner Oepen le 21.1.2009; Bernard DÉzERT, La croissance industrielle et urbaine de la porte d'Alsace. Essai géographique sur la formation d'un espace régional en fonction de l'attraction industrielle, Paris, Société d'Édition d'Enseignement Supérieur, 1969, p. 265. 


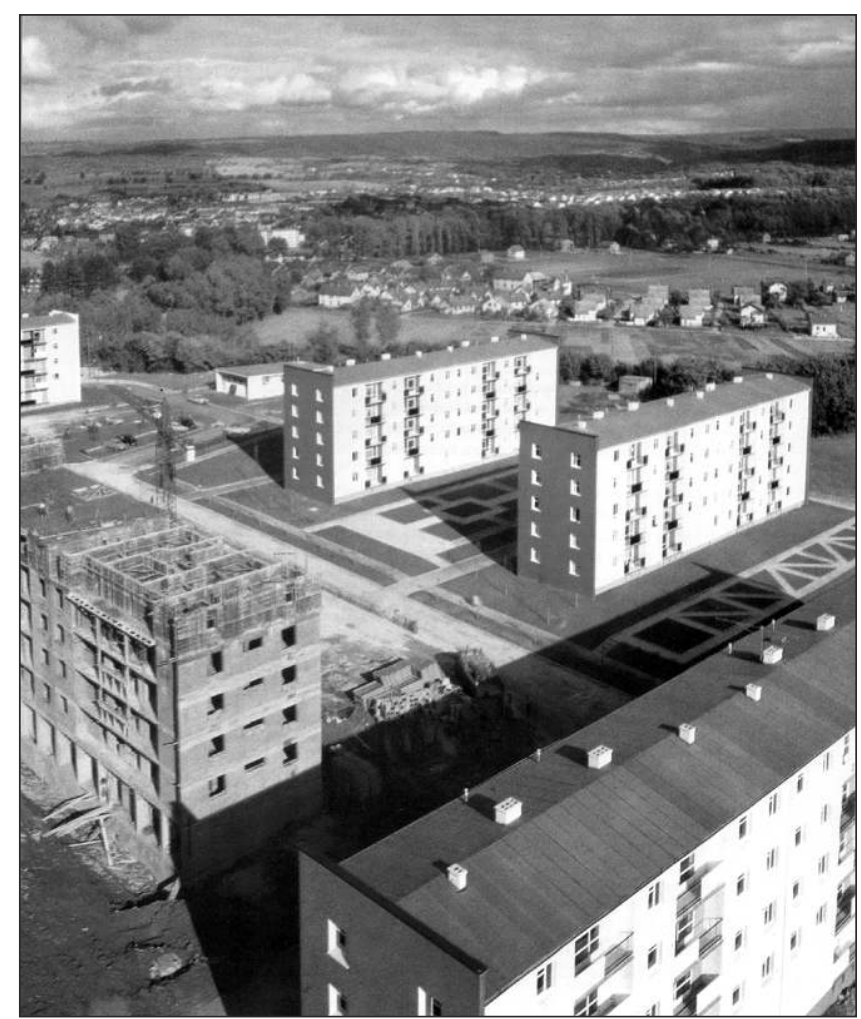

Figure 3: Le domaine des Buis près de Sochaux, 1959. MP. celui du logement pour célibataire, devenu une solution transitoire ${ }^{(33)}$. À Peugeot-Sochaux, le pourcentage de salariés vivant en logements pour célibataire est passé à $6,2 \%$ en 1963 , tandis que celui des logements d'entreprise est passé de 12,5\% fin 1951 à $18,6 \%$ à la fin de l'année 1963. L'usine avait plus de 4700 habitations locatives à sa disposition (figure 3) $^{(34)}$.

Chez Volkswagen, le pourcentage de salariés pouvant vivre en logements d'entreprise est passé de $6,3 \%$ à $18,6 \%$; cela correspondait à plus de 10000 logements (figure 4) ${ }^{(35)}$. Ce type de financement a contribué à diminuer la pénurie de logements et à en améliorer la qualité( ${ }^{(36)}$. Les différences quantitatives entre les deux entreprises sont liées à plusieurs raisons : avant de construire, il fallait assurer le financement, avoir des terrains et mobiliser l'infrastructure nécessaire pour de nouvelles cités.

La plupart des salariés des deux établissements souhaitaient construire leur propre maison, mais sans en avoir généralement les moyens. L’augmentation des salaires réels et les prêts d'entreprise leur en donnaient cependant davantage la possibilité. Bien que ces prêts limitent la liquidité des deux entreprises, ils ne représentaient pour elles qu’une dépense restreinte.

33 Nicolas Hatzfeld, Laure Pitti, «Usine et ville industrielle. Boulogne-Billancourt et PeugeotSochaux, entre face-à-face et échange», in: Danièle Rousselier-Fraboulet (dir.), La ville sans bornes, la ville et ses bornes, Paris, Nolin, 2006, p. 27-41, ici p. 31 sq.

34 AP Sochaux, Rapport technique, janvier 1953, graphique 39, in: Musée de l'Aventure Peugeot (MP), 34/65, et décembre 1963, graphique 45, in : MP, 34/82.

35 VWW, Jahresberichte des Personalwesens 1951-1952, 1963, in: UVW, Z 69 n 701.

36 Gerold Ambrosius, Hartmut Kaelble, «Einleitung. Gesellschaftliche und wirtschaftliche Folgen des Booms der 1950er und 1960er Jahre», in: Hartmut Kaelble (dir.), Der Boom 1948-1973. Gesellschaftliche und wirtschaftliche Folgen in der Bundesrepublik Deutschland und in Europa, Opladen, Westdeutscher Verlag, 1992, p. 7-32, ici p. 20. 
Les entreprises subventionnaient aussi le transport des salariés vers leur lieu de travail. Les besoins à Sochaux étaient plus grands ${ }^{(37)}$ que dans la région de Wolfsburg qui a pu profiter d'une bonne desserte ferroviaire et routière et d'une rapide mise en place de transports en commun. $49,3 \%$ des salariés à Sochaux utilisaient les cars subventionnés par Peugeot en septembre 1964; ensuite, ce pourcentage a diminué progressivement en raison du développement de l'automobile. La politique d'infrastructure a été primordiale pour l'expansion des effectifs pendant la deu-

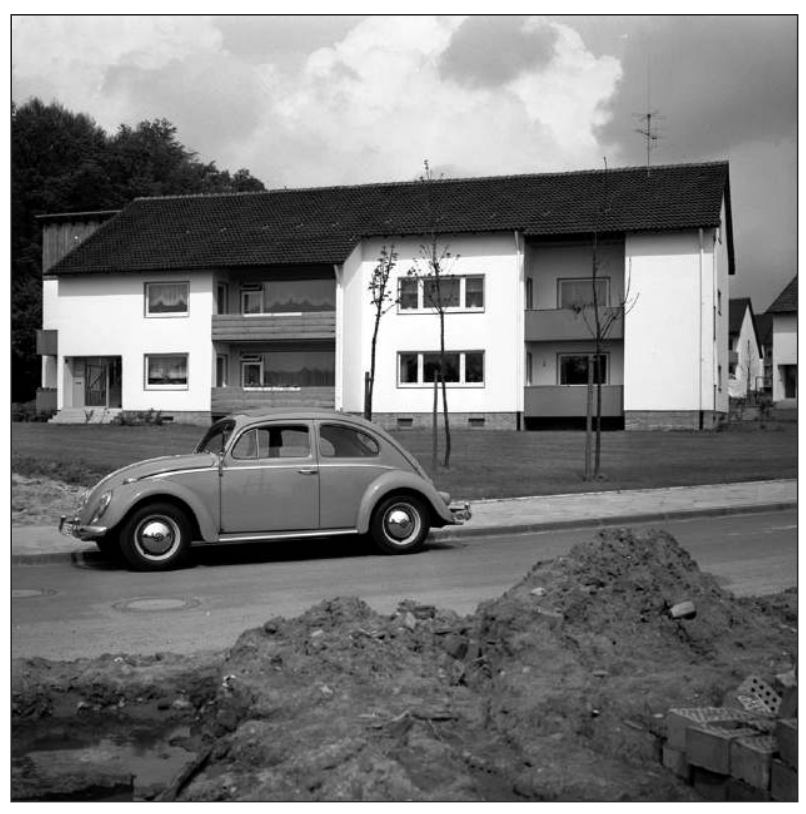

Figure 4: Logements de la Société d'habitations Volkswagen à Wolfsburg, 1961. Volkswagen Aktiengesellschaft, B61_248. xième période. Sans les logements et les cars d'entreprise, le recrutement d'effectifs aussi importants n'aurait pas pu être réalisé, en particulier à Sochaux.

Les activités hors entreprise ont aussi été valorisées pendant cette deuxième période et la politique liée aux congés a été marquée par une augmentation des destinations et du nombre de participants. En dehors des colonies de vacances, depuis le début des années 1960, le CE subventionnait de plus en plus les vacances familiales, en proposant des séjours au camping ou dans des locations individuelles. Volkswagen notamment a élargi son offre en matière de destinations de vacances. Mais l'engagement des deux entreprises dans le secteur des loisirs est resté différent. Volkswagen ne s'engageait que dans des cas exceptionnels et pour certains groupes cibles, tandis que le CE de Sochaux a maintenu les activités reprises de Peugeot. Au début des années 1960 en particulier, il a créé des clubs au sein de la nouvelle Association culturelle du personnel, dans un contexte comparable à celui de Renault et d'autres grandes entreprises ${ }^{(38)}$. L'importance de la politique des loisirs, bien que peu mise en avant par les deux entreprises, est donc un fait incontestable, dans un environnement peu favorable, aussi bien à Sochaux-Montbéliard qu'à Wolfsburg, alors que d'autres entreprises s'étaient détournées de ce type de politique ${ }^{(39)}$. Chez Peugeot, la politique des vacances en particulier semblait importante pour des raisons de concurrence ${ }^{(40)}$.

37 B. DÉZERT, La croissance industrielle et urbaine (note 32), p. 264 sq.

38 Jean-Pierre Le Сrom, L'introuvable démocratie salariale. Le droit de la représentation du personnel dans l'entreprise (1890-2002), Paris, Syllepse, 2003, p. 76 sq.

39 Christian Lamy, "Autour de Michelin. Mémoires», in: A. Gueslin (dir.), Les hommes du pneu (note 2), p. 293-329, ici p. 323.

40 N. Hatzfeld, Les gens d'usine (note 6), p. 212. 
La deuxième période est donc marquée par la croissance des prestations sociales dans les deux entreprises ${ }^{(41)}$, croissance qui a souvent surpassé l'augmentation des effectifs, une évolution qui montre l'importance particulière accordée aux prestations sociales dans les deux cas. Ayant établi les bases de leur système social jusqu'au début des années 1950, celles-ci ont diversifié les prestations, les ont augmentées et étendues à un plus grand nombre de salariés, plutôt que d'en créer de nouvelles ${ }^{(42)}$. L'une des rares exceptions a été l'engagement culturel du CE de Sochaux. L'ensemble de ces mesures représentait des avantages financiers importants pour les salariés. De leur côté, les entreprises avaient pour objectif une amélioration de la productivité. Progressivement, l'assistance et l'approvisionnement en biens matériels ont été relégués au second plan et les entreprises se sont concentrées sur le soutien en cas d'urgence ainsi que sur le versement d'allocations pour la majorité des salariés. Ni les vacances, ni la participation aux bénéfices n'ont eu l'importance qu'a prise la construction de logements en location pour le recrutement de la main-d'œuvre. Mais Peugeot et Volkswagen n'auraient pas profité de ces mesures si les nouveaux salariés avaient quitté l'entreprise rapidement. Au-delà du recrutement et de la volonté d'attacher les salariés à l'entreprise, les constructeurs ont aussi tenté d'améliorer leur motivation grâce à une participation au succès de l'entreprise. Même si l'efficacité économique des prestations sociales reste contestée, les directions en étaient convaincues, surtout dans les domaines du logement et de la participation financière, pour lesquels ils ne devaient guère prendre en compte les désirs des représentants du personnel. Ainsi, il nous semble que les mesures sociales d'entreprise ont constitué une condition de taille pour la réussite économique des constructeurs.

\section{Crises des ventes et nouvelles attentes - du milieu des années 1960 à la fin des années 1970}

Au milieu des années 1960, Peugeot et Volkswagen sont confrontées plus intensément à la volatilité de la demande automobile. Ceci les incite à ne pas laisser croître les prestations sociales dans une proportion plus grande que celle de la productivité( ${ }^{(43)}$. En 1973 et 1979, l'industrie automobile fait face aux chocs pétroliers. Chez Volkswagen, le premier choc se joint à une crise des ventes entre 1971 et 1975 . Les effectifs sont alors réduits de 13000 salariés. Peugeot est moins touchée par la crise de 1973/74, mais, à la suite du rachat de Chrysler Europe, le second choc pétrolier l'affecte plus durement. Entre 1978 et 1985, l'effectif de l'usine de Sochaux diminue de $30 \%{ }^{(44)}$.

Entre 1964 et 1979, Peugeot triple sa production qui atteint 865000 véhicules en 1979. En revanche, Volkswagen n'élève pas sa production de façon significative; pourtant, le personnel de l'usine de Wolfsburg a augmenté de $25,2 \%$, contre $50 \%$ environ pour

41 J.-L. Loubet, Automobiles Peugeot (note 6), p. 237.

42 Hans Günter Hockerts a déjà constaté ces tendances pour la politique sociale publique. ID., «Sozialpolitik in der Bundesrepublik Deutschland», in: H. PoнL (dir.), Staatliche, städtische, betriebliche und kirchliche Sozialpolitik (note 1), p. 359-379, ici p. 360.

43 Werner Abelshauser fait la même observation pour la BASF. ID., «Die BASF seit der Neugründung von 1952 », in: ID. (dir.), Die BASF. Eine Unternehmensgeschichte, Munich, Beck, 2003 (2éd.), p. 359-637, ici p. 417.

44 N. Hatzfeld, Les gens d’usine (note 6), p. 558; J.-L. Loubet, «PSA Peugeot Citroën» (note 6), p. 125 sq. 
celui de l'usine de Sochaux. Les salaires réels qui avaient fortement évolué pendant la deuxième période continuent à augmenter: Volkswagen les majore de 3,7\% par an en moyenne entre fin 1963 et $1975^{(45)}$, Peugeot de $1,8 \%$ entre fin 1963 et 1970 et d'environ $3 \%$ par an pendant les années $1970^{(46)}$. Chez Volkswagen, le versement du salaire en cas de maladie pendant six semaines était déjà en grande partie acquis en 1955, tandis que Peugeot payait $80 \%$ du revenu du $15^{\mathrm{e}}$ au $45^{\mathrm{e}}$ jour de maladie ${ }^{(47)}$. La mensualisation de la plupart des travailleurs à partir de 1970 leur donne aussi le droit à $100 \%$ de leur salaire.

Compte tenu des évolutions dues à la crise, on peut se demander si les constructeurs automobiles ont tiré parti des difficultés financières pour diminuer le niveau de leurs prestations sociales. Ou bien ont-ils plutôt aligné leurs politiques sociales sur les nouvelles conditions résultant des chocs pétroliers et les attentes nouvelles de leurs salariés? Dans cette troisième période, les deux entreprises sont moins disposées à introduire des prestations obligatoires; cependant, elles ne respectent plus nécessairement la maxime d'économie pendant les périodes de redémarrage économique, comme dans le cas du financement d'HLM. En même temps, la réglementation applicable aux entreprises et surtout le pouvoir de négociation des syndicats s'accroissent, de sorte que les entreprises ne peuvent pas empêcher une hausse des charges sociales. Chez Volkswagen, les dépenses supplémentaires s'élèvent à 33,8\% des salaires en 1980 , l'ensemble des charges sociales en représentant $61,5 \%{ }^{(48)}$. Chez Peugeot, avec $38,3 \%$, les charges sociales représentent également un pourcentage plus élevé de la masse salariale par rapport à 1966. Ces observations montrent que la croissance la plus importante des dépenses concernant les charges sociales s'observe pendant la troisième période.

En ce qui concerne l'approvisionnement en biens matériels et l'assistance, les priorités ont été maintenues, mais le volume financier des anciennes prestations a baissé. En outre, Peugeot et le CE de Sochaux ont diminué la concession de denrées. Ces réductions ne résultent pas des crises financières: les entreprises cessent des mesures qui ne leur semblent plus nécessaires. Les secours servent à de moins en moins de salariés. Initialement destinés aux cas d'urgence, les fonds de caisses de secours sont employés pour couvrir des dépenses «normales» telles que les soins dentaires. Les directions de Peugeot et le Betriebsrat se révèlent plus conservatrices que le management de Volkswagen et le CE de Sochaux. Ces attitudes divergentes sont fondées sur le fait que le CE pouvait réemployer des économies faites dans certaines institutions sociales pour en créer d'autres et disposer d'un budget indépendant depuis 1966, tandis que chez Volkswagen, cet argent revenait au management.

Les deux entreprises considèrent les prestations soumises aux conditions économiques comme porteuses d'espoir, favorables à la production et permettant de récompenser les

45 L'aperçu se termine en 1975. VWW, Durchschnittlicher Stundenlohn zum Jahresende in Pfennig, in: UVW, Z $119 \mathrm{n}^{\circ} 7$.

46 De 1971 à 1974, le salaire horaire moyen augmente de 3,7\% par année; de 1977 à 1980, le revenu mensuel d'un ouvrier de fabrication ou de maintenance augmente de 3,0 \%. Propres calculs selon AP Sochaux, Rapport technique 1964-1970, in: MP, 34/83 sq.

47 AP Sochaux, Rapport technique, Avril 1960, p. 20, in: MP, 34/79.

48 Le calcul des charges sociales est modifié en 1980 selon le rapport social de 1981, p. 13, sans préciser les modalités. 
salariés fidèles. Une autre raison de leur persistance tient au fait qu'elles étaient souvent fixées par des accords d'entreprise, des conventions collectives ou des lois donnant des droits aux salariés, ce qui était plutôt contraire aux intentions de la direction. De nombreuses prestations financières ne distinguent plus nos deux entreprises des autres du secteur, mais semblent nécessaires pour des raisons de concurrence (figure 5) ${ }^{(49)}$. Les syndicats chez Peugeot s'alignent souvent sur ceux de Renault, même si la direction rejette une telle comparaison ${ }^{(50)}$. Des mesures pour la protection des salariés sont modifiées si cela semble nécessaire pour satisfaire les partenaires sociaux: les retraites complémentaires ont été augmentées à partir de la fin des années 1960 comme celle de la Sécurité sociale ${ }^{(51)}$. Les constructeurs indemnisent aussi partiellement les salariés qui ne peuvent plus maintenir leurs postes de travail convenablement rémunérés. La mise en valeur des suggestions d'amélioration pourrait laisser penser que leur utilité économique gagne en importance, alors qu'en réalité les entreprises ne dépensaient pas plus d'argent pour les primes dans ce domaine. Comparées aux gratifications qui étaient allouées sans prise en compte de la performance, les dépenses pour les primes de suggestions sont assez faibles chez Volkswagen.

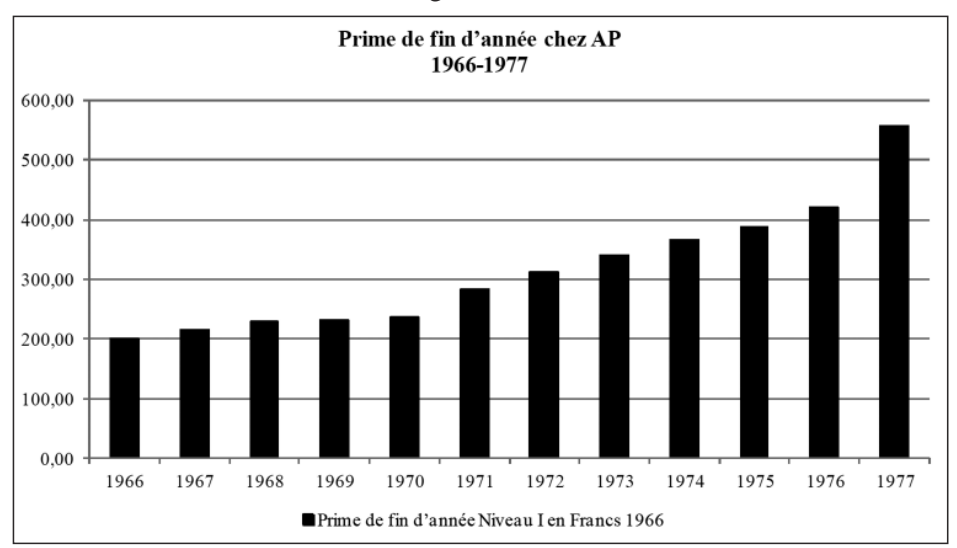

Figure 5: AP Sochaux, Rapport technique 1966-1970; JIP 1971-1977, in: MP.

Dans la troisième période, les prestations soumises à des critères économiques sont arrivées au premier plan de la politique sociale, surtout pendant les crises, car les entreprises tentent ainsi de motiver les salariés et d'élever leur productivité. Cet objectif est souvent voué à l'échec à cause de la rigidité croissante de ces prestations. En général, les réductions de prestation ont été limitées à court terme, le démantèlement social ne commençant réellement que dans les années $1980^{(52)}$.

49 Cf. entre autres VWW, Vorlage 2 FIP und ZPS zur Vorstandssitzung am 16.11.1976, 11.11.1976, p. 2, in: UVW, Z $373 \mathrm{n}^{\circ}$ 191/3; Treffen der Personalleiter der Automobilindustrie am 13. und 14.10.1977, in: UVW, Z $797 \mathrm{n}^{\circ} 1166$.

50 AP, PV de la réunion du CE de Sochaux le 17.11.1967, p. 77, et le 19.01.1971, p. 4 sq., in: Archives du CE de Sochaux à l'intérieur de l'usine (CE1), PV du CE.

51 Ambrosius/ Kaelble, «Einleitung» (note 36), p. 29.

52 Entretien avec Werner Oepen le 21.01.2009; René LAsserre, «Mitbestimmung und Betriebsverfassung in Deutschland und Frankreich. Elemente eines historischen und soziologischen Vergleichs", 
Pour le financement de logements et de maisons individuelles, Volkswagen met à disposition 347 millions de marks entre 1964 et 1976. Peugeot dépasse son obligation du $1 \%$ logement par trois fois au début des années $1970^{(53)}$. Néanmoins, ces dépenses n'ont pas réduit les bénéfices des entreprises dans les mêmes proportions car la construction subventionnée par les entreprises a toujours été aidée par l'État sous forme de prêts, subsides et avantages fiscaux ${ }^{(54)}$. En outre, les prêts des entreprises sont à rembourser ${ }^{(55)}$. Ainsi, les constructeurs ont fait des économies d'impôt pendant les années à fort bénéfice en effectuant des dépenses pour le financement de logements. En revanche, en temps de difficultés financières, elles ont diminué ces coûts, d'autant plus que ceux-ci ne semblaient plus nécessaires compte tenu de la réduction des effectifs. Le taux croissant de subventions versées aux sociétés extérieures de logement démontre la tendance à ne plus s'engager dans des mesures hors entreprise qu'en fonction des besoins, ce que les constructeurs précisent explicitement ${ }^{(56)}$. Ce qui avait semblé indispensable pour des besoins de rattrapage et pour l'expansion rapide des effectifs a désormais pris une connotation négative: depuis le milieu des années 1960, les entreprises essuient de plus en plus le feu de la critique sociétale à ce propos ${ }^{(57)}$. En outre, l'inflation et la réduction des avantages fiscaux ont enchéri le coût de construction. De plus en plus de salariés sollicitent également des prêts dans ce domaine. Dans ce contexte, la préférence des entreprises pour l'accession à la propriété ne surprend pas.

Les offres de vacances sont l'objet de négociations entre les managements et les CE qui tentent d'accorder une plus grande liberté aux salariés. Volkswagen n’a pas supprimé toutes ses maisons de vacances pendant les années $1970^{(58)}$, mais la transformation possible d'un séjour de convalescence en contribution financière a facilité à terme cette suppression. Bien que le constructeur ait renoncé à vouloir régir le temps libre de ses salariés, il a satisfait les attentes sociétales en fonctionnant comme mécène d'institutions culturelles et sociales. Le fait qu'il comptabilisait les dépenses pour ces subventions parmi les "prestations sociales supplémentaires» laisse entendre que celles-ci constituaient pour lui un devoir. À Sochaux, la majorité CGT/CFDT du CE a privilégié des investissements en vacances familiales ainsi que la subvention pour l’Association culturelle qui, avec 3,5\% du budget de 1970, était toutefois restreinte ${ }^{(59)}$.

in: Hans Poнl (dir.), Mitbestimmung und Betriebsverfassung in Deutschland, Frankreich und Großbritannien seit dem 19. Jahrhundert, Stuttgart, Steiner, 1996, p. 23-40, ici p. 39; J.-L. Loubet, Automobiles Peugeot (note 6), p. 398; VWW, Rapport annuel 1987, p. 41.

53 AP, PV de la réunion du CE de Sochaux 25.02.1972, p. 29 sq., in: CE1, PV du CE.

54 Bruno Lefebvre, Michel Moulllart, Sylvie Ochipinti, Politique du logement. 50 ans pour un échec, Paris, L'Harmattan, 1992, p. 27 sq.; Georg WAGNer, Sozialstaat gegen Wohnungsnot. Wohnraumbewirtschaftung und sozialer Wohnungsbau im Bund und in Nordrhein-Westfalen 1950-1970, Paderborn, Schöningh, 1995, p. 108 sq., p. 145.

55 VWW, Niederschrift über die Sitzung des Sozial-Ausschusses des Aufsichtsrats am 5.2.1963, in: UVW, Z $373 \mathrm{n}^{\circ} 785 / 2$.

56 AP, PV de la réunion du CE de Sochaux le 29.12.1969, p. 35, et le 28.06.1979, p. 185, in: CE1, PV du CE; VWW, Protokoll der Vorstandssitzung am 22.1.1965, p. 8, in: UVW, Z 373 n 455/1.

57 Werner Plumpe, «1968 und die deutschen Unternehmen. Zur Markierung eines Forschungsfeldes », Zeitschrift für Unternehmensgeschichte, vol. 49 (2004), p. 45-66, ici p. 51.

58 VWW, Protokoll der Vorstandssitzung am 4./5.03.1975, p. 11, in: UVW, Z 373 n $^{\circ}$ 187/2.

59 AP, PV de la réunion du CE de Sochaux le 22.04.1970, p. 41 sq., in: CE1, PV du CE. 
Les politiques de vacances et de loisirs montrent une libéralisation et une diversification des destinations et des activités. Pourtant, la demande en colonies de vacances diminue. Les salariés continuent à solliciter des subventions de congés, mais davantage avec leurs familles. Les loisirs traditionnels sont moins demandés que les offres nouvelles telles que les vacances à la neige.

Ayant été marquées par une expansion quantitative au cours de la seconde période, les politiques sociales des entreprises sont davantage soumises à des transformations qualitatives entre le milieu des années 1960 et la fin des années 1970. Cette évolution correspond aussi bien à une disposition moindre des entreprises à prendre des responsabilités à long terme sans y être obligées, étant donné la nécessité de faire des économies ${ }^{(60)}$, qu'aux attentes des salariés eux-mêmes. Même si Peugeot et Volkswagen se sont retirées de certains secteurs sociaux, ces décisions ne sont pas forcément liées aux crises économiques. Néanmoins, celles-ci facilitent la justification de décisions sociales restrictives. La majorité des mesures d'économies mises en place pendant les crises s'achevait dès qu'on apercevait la fin du tunnel. Souvent, les entreprises ne pouvaient pas faire prévaloir leurs objectifs d'économie car les syndicats et les salariés résistaient et obtenaient de plus en plus de droits aux prestations sociales. La progression de la propension au conflit qui s'est reflétée dans les événements de $1968^{(61)} \mathrm{a}$ eu des impacts différents chez Volkswagen et chez Peugeot: alors que le constructeur français tente d'esquiver des négociations avec la CGT et la CFDT, le management de Volkswagen intègre davantage l'IG Metall dans les politiques d'entreprise ${ }^{(62)}$.

À différents égards, Volkswagen et Peugeot ont élargi leurs politiques sociales de leur propre initiative, mais moins pour les familles que pour les salariés eux-mêmes. Par exemple, les constructeurs automobiles ont favorisé l'amélioration des conditions de travail en relation avec l'esprit du temps. Un autre accent est mis sur la protection des salariés fidèles. Les deux entreprises soulignent également l'importance des suggestions d'amélioration, mais celles-ci leur apportent un avantage économique direct et la participation des salariés ne suit pas leur développement. La politique sociale d'entreprise reflète différentes évolutions de la société. L'individualisation et la diversification des styles de vie passent pour des éléments marquants de la fin des années 1960 et des années $1970{ }^{(63)}$. Dans tous les domaines, nous avons trouvé des indicateurs d'une personnalisation des prestations sociales comme l'ont également constaté d'autres chercheurs ${ }^{(64)}$.

60 VWW, Protokoll der Vorstandssitzung am 7.6.1967, p. 12, in: UVW, Z 373 n 453/2 ; CA, n 172 , aoûtseptembre 1966, p. 2 sq., in: MP.

61 Emmanuel Quenson, «La gestion de personnel», in: Michel Freyssenet, Patrick Fridenson, JeanMarc Pointet (dir.), Les données économiques et sociales de Renault. Les années 70 et 80, Paris, GERPISA, 1995, p. 164-178, ici p. 164.

62 M. Durand, Grain de sable sous le capot (note 6), p. 410; Manfred Grieger, "Der neue Geist im Volkswagenwerk. Produktinnovation, Kapazitätsabbau und Mitbestimmungsmodernisierung», in: Morten Reitmayer, Ruth Rosenberger (dir.), Unternehmen am Ende des "goldenen Zeitalters". Die 1970er Jahre in unternehmens- und wirtschaftshistorischer Perspektive, Essen, Klartext (Bochumer Schriften zur Unternehmens- und Industriegeschichte, 16), 2008, p. 31-66, ici p. 56.

63 Ulfert Herlyn, Gitta Scheller, Wulf Tessin, Neue Lebensstile in der Arbeiterschaft? Eine empirische Untersuchung in zwei Industriestädten, Opladen, Leske + Budrich, 1994, p. 12, 238; Christian LE BART, L'individualisation, Paris, Presses de FNSP, 2008, p. 153 sq., p. 201.

64 N. Hatzfeld, Les gens d'usine (note 6), p. 385; Pierre Morville, Les nouvelles politiques sociales du patronat, Paris, La Découverte, 1985, p. 8 sq. 


\section{Conclusion}

Nous avons démontré que les systèmes sociaux des entreprises après 1945 n'étaient pas figés, mais qu'ils ont changé à beaucoup d'égards de la fin des années 1940 à la fin des années 1970. Nous pouvons décrire cette transformation comme une expansion des mesures sociales qui a eu lieu parallèlement à la progression des prestations sociales publiques ${ }^{(65)}$ et aux évolutions dans la société. Nous avons identifié trois processus de transformation en partie complémentaires.

Premièrement, les entreprises se sont retirées de plus en plus des œuvres sociales au profit d'une professionnalisation des prestations. Cela veut dire qu'elles ont préféré soutenir financièrement les offres proposées par des acteurs externes ou par les salariés plutôt que de proposer des offres elles-mêmes. Elles ont progressivement considéré l'apport de prestations non directement indispensables à la production comme subsidiaire. Ainsi, elles ont supprimé les prestations lorsque celles-ci ne semblaient plus nécessaires ou attractives ${ }^{(66)}$. Déjà apparue au cours de la première période, cette tendance s'est affirmée au cours de la troisième. Toutefois, le CE de Sochaux s'est distingué par sa politique de vacances et de loisirs.

Deuxièmement, l'accessibilité et la fiabilité des prestations sociales d'entreprise se sont accrues parallèlement aux prestations publiques ${ }^{(67)}$. De plus en plus, les salariés ont obtenu des droits aux prestations grâce à des accords d'entreprise, des conventions collectives et des lois. Nous pouvons discerner une tendance à l'augmentation des prestations sauf dans l'assistance et l'approvisionnement en biens matériels. Les entreprises ont aussi élargi le cercle des bénéficiaires des prestations sociales, mais avec une standardisation de ces dernières, déjà visible au cours de la première période.

Troisièmement, le nombre d'offres pour les salariés a augmenté. Cela s'est traduit par une monétarisation, c'est-à-dire le remplacement de prestations matérielles par un soutien financier. La progression des prestations soumises aux conditions économiques confirme cette tendance ${ }^{(68)}$. En outre, nous avons démontré la diversification des prestations existantes au cours de la seconde période. Ensuite, les entreprises ont aligné leurs prestations sociales de plus en plus sur les besoins de chaque salarié. Cela confirme une individualisation qui était déjà apparue au début des années 1960.

En ce qui concerne les prestations économiques, Volkswagen les garantissait plus tôt et à plus long terme, tandis que Peugeot poursuivait une stratégie de stimulation, lui accordant une plus grande flexibilité en temps de crise. Le constructeur français s'est

65 Alain BARJot (dir.), La Sécurité sociale. Son histoire à travers les textes, vol. 3, Paris, Association pour l'Étude de l'Histoire de la Sécurité sociale, 1988, p. 76, 83, 896; Manfred G. Scнмidt, Sozialpolitik in Deutschland. Historische Entwicklung und internationaler Vergleich, Wiesbaden, VS, 2005 (3éd.), p. 193 sq.

66 André Gueslin, «Introduction», in: ID. (dir.), Les hommes du pneu (note 2), p. 7-15, ici p. 15.

67 Günther Schulz, «Armut und soziale Sicherung. Zwischen Versorgung und Versicherung», in: Reinhard Spree, Rolf CAESAR (dir.), Geschichte der deutschen Wirtschaft im 20. Jahrhundert, Munich, Beck, 2001, p. 157-177, ici p. 171.

68 Roland Reichwein, Funktionswandlungen der betrieblichen Sozialpolitik. Eine soziologische Analyse der zusätzlichen betrieblichen Sozialleistungen, Cologne et al., Westdeutscher Verlag, 1965, p. 89, $169 s q$. 
aussi engagé à une protection croissante des revenus. Des priorités différentes sont également à observer dans la politique d'infrastructure: Volkswagen a privilégié la construction de logements tandis que Peugeot a investi dans les cars d'entreprise, pour des raisons financières et régionales. Le respect d'une certaine tradition chez Peugeot explique aussi le maintien de prestations considérées ailleurs comme démodées comme les cadeaux de Noël. Mais Volkswagen et Peugeot fournissaient finalement des prestations sociales assez semblables en dépit des divergences des législations française et allemande sur la cogestion, ce qui montre que l'influence du secteur était grande.

Nous avons démontré que la politique sociale d'entreprise est devenue de plus en plus un objet de négociation entre différents acteurs. Les managers étaient de moins en moins en mesure de modifier des prestations sociales sans concertation avec les syndicats car ils risquaient alors la grève, assez probable en raison du taux élevé de syndicalisation. La judiciarisation contribuait aussi à l'intégration d'acteurs supplémentaires. Le rôle du CE de Sochaux ne saurait être moindre que celui du Betriebsrat chez Volkswagen, car le CE était très actif dans le domaine social et gérait les œuvres sociales de manière assez autonome depuis 1966. Cela étant, il y avait des minorités syndicales plus fortes au CE qu'au Betriebsrat depuis les années 1950, de sorte que la majorité CGT/CFDT formée depuis 1961 avait plus de mal à s'imposer que l'IG Metall. Alors que les représentants du personnel français acceptaient des réductions des prestations sociales, le Betriebsrat s'opposait souvent aux changements. La raison principale en était que le CE de Sochaux disposait d'un budget fixe depuis 1966, tandis que son homologue de Volkswagen devait le négocier. Cela rendait pour ce dernier la réalisation de nouvelles mesures sociales plus difficile car il devait en convaincre la direction.

Notre étude a montré que l'établissement de la politique sociale d'entreprise après 1945 ne peut s'expliquer que grâce à de nombreux facteurs et qu'il ne faut pas l'associer uniquement à l'intention d'une entreprise de «faire du bien» ou de recruter et de fixer la main-d'œuvre. Pour finir, nous allons mettre en valeur les principaux de ces facteurs. L'emplacement géographique des usines de Sochaux et de Wolfsburg a rendu d'abord nécessaire un certain nombre de mesures sociales. Celles-ci ne pouvaient être réalisées que si la situation financière des entreprises le permettait. Par ailleurs, la politique sociale d'entreprise était aussi censée concourir à la réduction des frais de personnel. Le manque de main-d'œuvre entre le début des années 1950 et celui des années 1970 a obligé les constructeurs à se positionner par rapport à leur secteur de production grâce à des prestations sociales spécifiques. Une autre raison pour l'amélioration des conditions sociales a été la satisfaction des salariés, de leurs représentants ainsi que des syndicats, qui ont gagné en influence. La législation a joué aussi un rôle pour l'ajustement ainsi que l'établissement de nouvelles prestations. De plus en plus, les attentes des consommateurs au sein de l'économie de marché sont devenues importantes. Dans le cas de Peugeot, le rôle de la structure familiale de l'entreprise et de la direction centrale du personnel a été souligné par des témoins de l'époque, mais n’a pu être exploré suffisamment faute d'avoir accès aux sources pertinentes. Compte tenu de la participation accrue d'autres acteurs, il nous semble cependant que l'on peut parler, à propos du Volkswagenwerk à Wolfsburg et d'Automobiles Peugeot à Sochaux pendant la période 1944-1980, d'une démocratisation de la politique sociale d'entreprise. 


\section{Résumé}

Contrairement à l'hypothèse de la perte d'importance de la politique sociale d'entreprise après 1945, de grandes entreprises comme le Volkswagenwerk et Automobiles Peugeot ont même élargi celle-ci. Certes, elles regardaient leurs prestations sociales comme étant subsidiaires et ont préféré soutenir les offres sociales d'autres initiateurs, mais malgré ceci, ils ont de plus en plus développé leurs prestations, les ont garanties et attribuées à plus de bénéficiaires. Par leur diversification, individualisation et monétarisation, les salariés ont obtenu plus de possibilités d'exercer de l'influence sur la politique sociale d'entreprise. De manière croissante, d'autres acteurs tels que les CE et les syndicats étaient impliqués dans la mise en ouvre de cette politique. Ainsi, malgré des processus temporairement opposés, il semble justifié de parler d'une démocratisation des prestations sociales d'entreprise après 1945.

\section{Zusammenfassung}

Entgegen der Hypothese, dass die betriebliche Sozialpolitik nach 1945 an Bedeutung verloren hat, bauten Großunternehmen wie das Volkswagenwerk und Automobiles Peugeot diese sogar aus. Zwar betrachteten sie ihre sozialen Leistungen als subsidiär und zogen es vor, die sozialen Angebote anderer Initiatoren zu unterstützen, aber dennoch erhöhten sie ihre Leistungen in zunehmenden Maße, machten diese verbindlicher und erweiterten den Empfängerkreis. Durch deren Diversifikation, Individualisierung und Monetarisierung erweiterten sich die Einflussmöglichkeiten der Beschäftigten auf die betriebliche Sozialpolitik. Auch andere Akteure wie die Betriebsräte und Gewerkschaften wurden verstärkt in die Gestaltung dieser Politik einbezogen. Insofern scheint es, trotz zeitweise rückläufiger Entwicklungen, gerechtfertigt, von der Demokratisierung der betrieblichen Sozialleistungen nach 1945 zu sprechen.

\footnotetext{
Abstract

In contrast to the hypothesis of the loss of importance of corporate social policy after 1945, big companies like the Volkswagenwerk and Automobiles Peugeot have even expanded it. Indeed, they considered their social measures as being subsidiary and preferred encouraging social offers of other initiators, but nonetheless, they increasingly raised their benefits, guaranteed them and granted them to more beneficiaries. The employees' possibilities to influence corporate social policy have grown through its diversification, individualisation and monetisation. Other stakeholders as the work councils and labour unions have been more and more involved in the shaping of corporate social policy, too. Thereby it seems justified, despite temporary opposing developments, to speak of the democratisation of corporate social policy after 1945.
} 